\title{
Locally Homogeneous and Isotropic Gaussian Fields in Kriging
}

\author{
Leonidas SAKALAUSKAS \\ Institute of Mathematics and Informatics, Vilnius University \\ Akademijos 4, 08663 Vilnius, Lithuania \\ e-mail:sakal@ktl.mii.lt
}

Received: April 2012; accepted: January 2013

\begin{abstract}
The paper deals with the application of the theory of locally homogeneous and isotropic Gaussian fields (LHIGF) to probabilistic modelling of multivariate data structures. An asymptotic model is also studied, when the correlation function parameter of the Gaussian field tends to infinity. The kriging procedure is developed which presents a simple extrapolator by means of a matrix of degrees of the distances between pairs of the points of measurement. The resulting model is rather simple and can be defined only by the mean and variance parameters, efficiently evaluated by maximal likelihood method. The results of application of the extrapolation method developed for two analytically computed surfaces and estimation of the position of the spacecraft re-entering the atmosphere are given.
\end{abstract}

Keywords: multivariate normal distribution, homogeneous Gaussian field, maximal likelihood, Wiener process.

\section{Introduction}

Decision making and recognition of data patterns in real-life problems often refer to model design using multidimensional data obtained by physical measuring or computational code. Such models arise in reconstruction of missing data, data extrapolation, experimental design or optimization with costly computed objective function, when the measurements or objective function values are available only partially, and forecasting according to historically observed data. Any physical or computer experiment usually brings many measures that may concern various quantities at a given time. No matter in what topics they are, also, no matter how they have been obtained, in a physical or computer experiment. The measure points, depending on a large number of parameters, may have been chosen at random, or in a deterministic way. The results themselves depend upon various parameters, and the choice and impact of these parameters are usually unclear. But conversely, the number of data that are collected is typically small, because each trial takes time and costs money. In some cases, the experiment may be dangerous, or the favourable circumstances are rare, in all cases, it is costly.

Real-life problems, dealing with such data modelling are numerous. For example, exploration of safety of a nuclear reactor in a critical state is related with extrapolation 
of measurement data obtained in steady functioning (Levenson and Rahn, 1981). In ecotoxicology, the toxicity experiments are usually done upon a small number of species, over a small number of days, and one wishes to extrapolate the results to a larger number of species and to longer periods, etc. (Solomon et al., 2008). The study of trajectography of a missile or spacecraft is both dangerous and quite costly, and requires forecasting according to the measured trajectory data. Experimental design in chemical engineering that aims to create new composite materials is often related with experiment planning using the data obtained from a series of experiments, etc.

The structures in such data are explored in traditional deterministic or statistical ways. However, the deterministic data analysis by interpolation or extrapolation uses some artificial assumptions and does not take into account the uncertainty related with data reconstruction (Shepard, 1968; Shumaker, 1976). Indeeds, the representation of experimental measuring or computer computation data is of a probabilistic nature, because, first, recovered data present only better or worse approximation and are uncertain before reconstruction, and second, the measurement or computation errors should be taken into account, too. Besides, the deterministic methods are quite inefficient when the dimensionality of the problem is high, although it is often the case in real-life problems. In turn, the statistical methods such as a regression and correlation analysis mostly describe the local properties of the data structure, say, linear or nonlinear trends, etc.

Following the considerations above, the concept of kriging has been developed dealing with the techniques of interpolating the value of a random field in an unobserved location from the observations of its value at nearby locations (Krige, 1951; Mattheron, 1963). Kriging computes the best linear unbiased estimator based on the probabilistic modelling of the results of observations or computations of a homogeneous random field, qualified by the expectation and the covariance function (Stein, 1999).

The Experimental Probabilistic Hypersurface (EPH) is a concept similar to kriging which has been discussed to meet a need connected with the representation of the information on the data obtained in some way (Beauzamy, 2004). EPH should give a way of anticipating, with some probability, a result associated with values of the parameters that have never been met before, or have been lost, to "store" the existing information (the measurements), and propagate it to any situation where no measurement has been made. Thus, the result of EPH should be a density of probability above each point of the configuration space (Beauzamy, 2004).

The paradigm of probabilistic modelling of the objective function with random fields in the optimization has been proposed and developed (Mockus, 1968, 1989; Jones, 2007). This paradigm has been appeared rather fruitful and found many applications in a Bayesian approach for global optimization, some heuristics for global search, extrapolation of functions with many variables, termination of stochastic algorithms, etc. (Mockus et al., 1997; Zilinskas, 1982; Bartkute and Sakalauskas, 2009; etc.).

Random Gaussian fields are widely met in probabilistic modelling. For instance, modelling using homogeneous and isotropic Gaussian fields (multivariate stationary processes) has found many applications in bioinformatics, engineering and physics, when data are observed in the experiment or computed by a certain computer code (Adler, 
1981; Bogush and Elkins, 1986; Goff et al., 1994; Chamon, 1996; Lopez-Caballero and Modaressi-Farahmand-Razavi, 2010; Zaichik and Alipchenkov, 2010; etc.). The properties of Gaussian field models depend in essence on the covariance function, qualifying the interdependence of points at which the measurement or computing experiments were performed. For instance, continuity or differentiability of the samples of random field depends on the continuity or differentiability of the covariance function, etc. Since the covariance function depends on the distance between the points of measurement or observation, the interrelations of multivariate data modeled with random Gaussian field are described by a matrix of pairwise distances among these points. Recently the matrices of squares of Euclidean distances have been explored best, however, matrices with arbitrary degrees of pairwise distances are not studied enough.

This paper analyses the properties of locally homogeneous and isotropic Gaussian fields (LHIGF) that are limiting for random fields covariance function of which can be expressed by the Taylor formula in the neighbourhood of zero point. The class presented has a transparent geometrical interpretation and enables us to model multivariate data, using the matrix of distances among the points of measurement or observation. The paper rests as follows: the LHIGF is introduced and discussed in the next section, asymptotic properties of kriging procedure are considered in Section 4 deriving new extrapolation formulas, the results of computer modelling and numerical application of the kriging procedure developed are presented in Sections 5 and 6.

\section{Locally Homogeneous and Isotropic Gaussian Fields}

Let us consider probabilistic modelling of a real-valued function or a response surface the values of which are obtained by observation and measurement or computing of a certain code, etc. Since there is no additional information except the fact of measurement performance at the experimental points, the surface that represents the objective function or response surface may be constructed as a homogeneous Gaussian field $Y(x, \omega), x \in \Re^{n}$, here $\omega \in(\Omega, \Sigma, P)$ is a random event in a probabilistic space (Matheron, 1963; Mockus, 1989; Jones, 2001). At this stage, all the parameters might be considered as equivalent, because it is unknown which ones will be preponderant. So a distance between the measurement points is taken, which is symmetric with respect to the various parameters, there are no weights. Hence, homogeneity and isotropy assumptions imply the surface under construction to be a sample of the Gaussian field with a constant mean vector

$$
E Y(x, w)=\mu
$$

constant variance

$$
E(Y(x, w)-\mu)^{2}=\sigma^{2},
$$

$\sigma^{2}>0$, and the correlation function

$$
E\left(Y\left(x^{\prime \prime}, \omega\right)-\mu\right) \cdot\left(Y\left(x^{\prime}, \omega\right)-\mu\right) / \sigma^{2}=\rho(r)
$$


depending on the distance between two points $\tau=\left|x^{\prime}-x^{\prime \prime}\right|$. The correlation function should necessarily be a positively defined function (Matern, 1986; Bisgaard and Sasvari, 2000). Of course, the correlation function of a homogeneous and isotropic Gaussian field also satisfies the natural assumptions: $|\rho(\tau)| \leqslant 1, \rho(0)=1$, and $\rho(\tau) \rightarrow 0$, as $\tau \rightarrow$ $\infty$. In the one-dimensional case a homogeneous Gaussian field becomes a stationary Gaussian process.

The sample properties of the homogeneous Gaussian field depend, in essence, on the correlation function. Say, it is well known that samples of the Gaussian field are continuous with probability one (almost surely, a.s.), if the correlation function is continuous in the neighborhood of zero, i.e., when $\lim _{r \rightarrow+0} \rho(r)=1$, (Adler, 1981; Pottgoff, 2010). Similarly, the samples of a random Gaussian field are differentiable, if the correlation function is twice differentiable (Pottgoff, 2010). Note, that the derivative of a random Gaussian field with respect to a certain component of $x$ is a random Gaussian field again covariance function of which is equal to the second derivative of the covariance function of the original field.

The exponential correlation function is often applied to probabilistic modeling using a random field:

$$
\rho(\tau)=e^{-\left(\frac{\tau}{\lambda}\right)^{\delta}},
$$

where $\gamma>0$ is a scaling parameter, $0<\delta \leqslant 2$. Hence, the samples of a random Gaussian field with the exponential correlation function are continuous a.s., as $\delta \geqslant 0$. Similarly, the samples of random Gauusian field with exponential correlation function (6) are differentiable a. s. with respect to $x$ components, as $\delta=2$. Conclusions on the continuity and differentiability of Gaussian field samples are very important, because the functions and response surfaces considered in multi-dimensional extrapolation, global optimization, etc., are mainly continuous or differentiable.

Note that if $n=1, \delta=1$, the resulting one-dimensional model with the exponential correlation function is a stationary Markov process, called as an Ornstein-Uhlenbeck process. Thus, the exponential function is a natural correlation in one dimension, since it corresponds to a Markov process. In two dimensions this is no longer so, although the exponential is a common correlation function in geostatistical work and kriging as well. Whittle (1954) determined the correlation corresponding to a stochastic differential equation of Laplace type noise, which describes a second order autoregression on a discrete lattice process (see, also Lim and Teo, 2009). Following this model, a class of the Whittle-Matern correlation function has been developed, presented by Goff and Jordan (1988) to parameterize the smoothness of realizations of the corresponding random field.

Let us confine ourselves to locally homogeneous and isotropic Gaussian fields (LHIGF) further, i.e., assume the homogeneity and isotropy assumptions to be valid in a certain convex bounded set $D \subset \Re^{n}$. Of course, homogeneous and isotropic random fields are locally homogeneous and isotropic ones, too. Assume, for simplicity, the set of local homogeneity and isotropy to be a ball $D \subset \Re^{n}$ of diameter $\operatorname{diam}(D)>0$. Thus, the requirements of constant mean (1), variance (2) and dependence of correlation (3) 
on the distance between the points of measurement of LHIGF are valid in the set of homogeneity and isotropy $D$. Note that LHIGF are not ergodic in general. The example of a non-ergodic locally stationary Markov process with independent increments is considered below.

EXAMPLE 1. Let us consider the sum of two Wiener processes

$$
W(x)=W_{+}(x)+W_{-}(x), \quad x \in \Re,
$$

where both Wiener processes $W_{+}(x)$ and $W_{-}(x)$ have the same parameter $\sigma^{2}>0$, start away from the ends of the interval $D=[-\gamma / 2, \gamma / 2]$ and continue to both sides of the real number axis. Then one can be sure that $W(x)$ is a locally stationary Gaussian process on the interval $D$ with mean $\mu=0$, variance $\sigma^{2} \cdot d$ and the correlation function $\rho(\tau)=1-\frac{\tau}{\gamma}$.

In fact, the expressions of variance

$$
E W^{2}(x)= \begin{cases}\sigma^{2} \cdot \gamma, & x \in D \\ 2|x| \cdot \sigma^{2}, & x \notin D\end{cases}
$$

and the covariance function:

$$
\begin{aligned}
E W & (x) \cdot W(y) \\
\quad & \begin{cases}\sigma^{2} \cdot(\gamma-|x-y|), & x, y \in D, \\
\sigma^{2} \cdot(\gamma / 2+\operatorname{sign}(x) \cdot y), & |x| \geqslant \gamma / 2, y \in D, \\
\sigma^{2} \cdot(\gamma / 2+\operatorname{sign}(y) \cdot x), & |y| \geqslant \gamma / 2, x \in D, \\
2 \cdot \sigma^{2} \cdot \min (|x|,|y|), & x \leqslant-\gamma / 2, y \leqslant-\gamma / 2 \text { or } x \geqslant \gamma / 2, y \geqslant \gamma / 2, \\
0, & x \leqslant-\gamma / 2, y \geqslant \gamma / 2 \text { or } y \leqslant-\gamma / 2, x \geqslant \gamma / 2,\end{cases}
\end{aligned}
$$

follows in an elementary way from the properties of Wiener processes. Independence of increments and Markovity of the process $W(x)$ follow from analogous properties of the processes $W_{-}(x)$ and $W_{+}(x)$.

\section{Kriging Using LHIGF}

The aim of kriging is to estimate the value of a real-valued function or response surface at a certain point by the values of the function given at some other points. Thus, assume to be given the data set of $K n$-dimensional points

$$
X=\left(x^{1}, x^{2}, \ldots, x^{K}\right),
$$

$x^{i} \in \Re^{n}, 1 \leqslant i \leqslant K$, and values of some response function

$$
Y=\left(y^{1}, y^{2}, \ldots, y^{K}\right)^{T},
$$


obtained at these points by means of physical measurement or simulation by computer, etc.

Let us briefly overview the kriging procedure under the assumption that the values of sample $Y$ be following from the homogeneous and isotropic Gaussian field $Y(x, \omega)$, defined by mean $\mu$, variance $\sigma^{2}$ and a certain correlation function $\rho(\tau)$. Thus, the dependences among points (9) are described by the correlation matrix:

$$
\Sigma=\left[\rho_{i j}\right]_{1}^{K},
$$

where $\rho_{i j}=\rho\left(\left|x^{i}-x^{j}\right|\right), 1 \leqslant i, j \leqslant K$.

Usually the parameters of random fields are calibrated by the maximal likelihood method. Maximal likelihood estimates (MLE) of mean and variance are as follows (Adler, 1981; Jones, 2001; Stein et al., 2004):

$$
\begin{aligned}
& \tilde{\mu}=\frac{T^{T} \cdot \Sigma^{-} \cdot E}{E^{T} \cdot \Sigma^{-1} \cdot E}, \\
& \tilde{\sigma}^{2}=\frac{(y-\tilde{\mu} \cdot E)^{T} \cdot(y-\tilde{\mu} \cdot E)}{K},
\end{aligned}
$$

where $E=(1,1, \ldots, 1)^{T}$ is the vector-row with $K$ components equal to $1, \Sigma>0$.

If the correlation function involves some other parameters, their MLE can also be obtained by respective maximization of the likelihood function.

Assume the vector of correlations between a certain point $x \in \Re^{n}$ and points of measurement (8) to be given: $\tau(x)=\left(\tau_{1}, \tau_{2}, \ldots, \tau_{K}\right)^{T}, \tau_{i}=\rho\left(\left|x_{i}-x\right|\right), 1 \leqslant i \leqslant K$. The predicted function value at this point is random, in general and distributed normally $N(y(x), s(x))$ with the mean equal to conditional expectation of the Gaussian field at the point $x$ :

$$
\begin{aligned}
y(x) & =E\left(Y(x, \omega) \mid Y\left(x_{1}, \omega\right)=y_{1}, Y\left(x_{2}, \omega\right)=y_{2}, \ldots, Y\left(x_{K}, \omega\right)=y_{K}\right), \\
y(x) & =\hat{\mu}+\tau(x)^{T} \cdot \Sigma^{-1} \cdot(Y-E \cdot \hat{\mu}) \\
& =Y^{T} \cdot \Sigma^{-1} \cdot\left(\tau(x)+E \cdot \frac{1-\tau(x)^{T} \cdot \Sigma^{-1} \cdot E}{E^{T} \cdot \Sigma^{-1} \cdot E}\right) .
\end{aligned}
$$

Variance of predictor (13) is equal to

$$
s^{2}(x)=\hat{\sigma}^{2} \cdot\left(1-\tau(x)^{T} \cdot \Sigma^{-1} \cdot \tau(x)+\frac{\left(1-r(x)^{T} \cdot \Sigma^{-1} \cdot E\right)^{2}}{E^{T} \cdot \Sigma^{-1} \cdot E}\right),
$$

where the first part on the right-hand side of (14) corresponds to the conditional variance and the last term can be interpreted as representing the uncertainty without knowing $\mu$ exactly, but rather having to estimate it from the data (Adler, 1981; Jones, 2001).

Denote the symmetric matrix of degrees of distances between the pairs of measurement points (8) by

$$
A=\left[r_{i j}^{\delta}\right]_{1}^{K}
$$


where $0<\delta \leqslant 2, r_{i j}=\left|x_{i}-x_{j}\right|, 1 \leqslant i, j \leqslant K$ and assume, the correlation function be satisfying the property:

$$
\rho(\tau)=1-\frac{a \cdot \tau^{\delta}}{\gamma^{\delta}}+O\left(\left(\frac{\tau}{\gamma}\right)^{2 \cdot \delta}\right)
$$

where $a=\left.\frac{d \rho(\tau)}{d \tau}\right|_{t=+0}, \gamma>0,0<\delta \leqslant 2$. For instance, the exponential function (4) presents an example of the correlation function, which met the latter assumption, as $a=1$. Thus, the correlation matrix can be represented now as:

$$
\Sigma=E \cdot E^{T}-\frac{a \cdot A}{\gamma^{\delta}}+\mathrm{O}\left(\gamma^{-2 \cdot \delta}\right)
$$

Theorem 1. Let the set of vectors $X=\left(x^{1}, x^{2}, \ldots, x^{K}\right), x^{i} \in \Re^{n}, 1 \leqslant i \leqslant K$, and set of values of a certain response function at these points $Y=\left(y^{1}, y^{2}, \ldots, y^{K}\right)^{T}$ be given, where the matrix of degrees of pairwise distances between points of measurement $A=\left[r_{i j}^{\delta}\right]_{1}^{K}, 0<\delta \leqslant 2, r_{i j}=\left|x_{i}-x_{j}\right|, 1 \leqslant i, j \leqslant K$, is non-singular: $|A| \neq 0$, and $E \cdot A^{-1} \cdot E^{T} \neq 0$. Assume that the values of measurements $Y$ follow to the homogeneous and isotropic Gaussian field having the mean $\mu$, the variance $\delta^{2}=d^{2} \cdot \gamma^{\delta} / a$ and the correlation function $\rho(\tau)$, which satisfies property (16).

Then, the asymptotic MLE's of field parameters are as follows:

$$
\begin{aligned}
& \hat{\mu}=\frac{y \cdot A^{-1} \cdot E^{T}}{E \cdot A^{-1} \cdot E^{T}}+\mathrm{O}\left(\gamma^{-\delta}\right) \\
& \frac{\hat{\delta}^{2} \cdot a}{\gamma^{\delta}}=\frac{1}{K} \cdot\left(\frac{\left(y^{T} \cdot A^{-1} \cdot E\right)^{2}}{E^{T} \cdot A^{-1} \cdot E}-y^{T} \cdot A^{-1} \cdot y\right)+O\left(\gamma^{-\delta}\right)
\end{aligned}
$$

The proof is presented in Appendix 1. It is based on the inverse with ShermanMorrison formula (Horn and Johnson, 1991):

$$
\left(E \cdot E^{T}-\frac{a \cdot A}{\gamma^{\delta}}\right)^{-1}=\frac{\gamma^{\delta}}{a} \cdot\left(\frac{A^{-1} \cdot E \cdot E^{T} \cdot A^{-1}}{E^{T} \cdot A^{-1} \cdot E-a / \gamma^{\delta}}-A^{-1}\right) .
$$

Let us denote $d^{2}=a \cdot \sigma^{2} / \gamma^{\delta}$. Then MLE of the parameter $d^{2}$ is given by (19). Hence, $\hat{\sigma}^{2}=\stackrel{\leftarrow 2}{d} \cdot \gamma^{\delta} / a$

Theorem 2. Let the conditions of Theorem 1 be valid. Besides, assume that $\tau(x)=$ $\left(\tau_{1}, \tau_{2}, \ldots, \tau_{K}\right)^{T}, \tau_{i}=\left|x_{i}-x\right|^{\delta}, 1 \leqslant i \leqslant K, 0<\delta \leqslant 2$, is the vector of degrees of the distances between a certain point $x \in \Re^{n}$ and points of the set of measurement $X$. The predicted function value at this point according to the kriging procedure (13), (14) is as follows

$$
y(x)=y^{T} \cdot A^{-1} \cdot\left(\tau(x)-E \cdot \frac{E^{T} \cdot A^{-1} \cdot \tau(x)-1}{E^{T} \cdot A^{-1} \cdot E}\right)+\mathrm{O}\left(\gamma^{-\delta}\right),
$$


the variance of which is:

$$
s^{2}(x)=\hat{d}^{2}\left(\frac{\left(E^{T} \cdot A^{-1} \cdot \tau(x)-1\right)^{2}}{E^{T} \cdot A^{-1} \cdot E}-\tau(x)^{T} \cdot A^{-1} \tau(x)\right)+\mathrm{O}\left(\frac{|\tau|^{\delta}}{\gamma^{\delta}}\right)
$$

The proof is given in Appendix 1, too.

Let us consider two univariate examples. The first one demonstrates the kriging for a locally stationary process with independent increments (see Example 1).

EXAMPLE 2. Let us consider a locally stationary Gaussian process with the mean, variance, and the correlation function $\rho(\tau)=1-\tau / 2$, defined on the interval $D=[-1,1]$ (see Example 1). Assume that the points of observations are arranged in an increasing order: $-1 \leqslant x^{1}<x^{2}<\cdots<x^{K} \leqslant 1$.

Now the correlation matrix looks like $E \cdot E^{T}-\frac{A}{2}$. It is easy to make sure that the inverse of the distance matrix $A=\left[\left|x^{i}-x^{j}\right|\right]_{1}^{K}$ is a modified tridiagonal matrix:

$$
\begin{aligned}
& A^{-1}=\frac{1}{2} \cdot\left[\begin{array}{cccc}
\frac{1}{x^{K}-x^{1}}-\frac{1}{x^{2}-x^{1}} & \frac{1}{x^{2}-x^{1}} & 0 & \ldots \\
\frac{1}{x^{2}-x^{1}} & \frac{-1}{x^{2}-x^{1}}-\frac{1}{x^{3}-x^{2}} & \frac{1}{x^{3}-x^{2}} & \cdots \\
0 & \frac{1}{x^{3}-x^{2}} & \frac{-1}{x^{3}-x^{2}}-\frac{1}{x^{4}-x^{3}} & \cdots \\
\cdots & \cdots & 0 & \cdots \\
\frac{1}{x^{K}-x^{K-1}} & \frac{1}{x^{K}-x^{K-1}} & & \\
\frac{1}{x^{K}-x^{1}} & 0 & 0 & \cdots
\end{array}\right. \\
& \left.\begin{array}{cc}
0 & \frac{1}{x^{K}-x^{1}} \\
0 & 0 \\
0 & 0 \\
\cdots & \cdots \\
\frac{-1}{x^{K-1}-x^{K-2}-\frac{1}{x^{K}-x^{K-1}}} & \frac{1}{x^{K}-x^{K-1}}
\end{array}\right]
\end{aligned}
$$

Using (18), (19) and (20), it is easy to make sure that MLE of parameters of the process considered are expressed in the following way:

$$
\begin{aligned}
& \hat{\mu}=\frac{y_{1}+y_{K}}{2} \\
& \hat{d}^{2}=\frac{1}{2 \cdot K} \cdot \sum_{i=1}^{K-1} \frac{\left(y_{i+1}-y_{i}\right)^{2}}{\left(x_{i+1}-x_{i}\right)} .
\end{aligned}
$$


Next, one can make sure that the kriging predictor of this process is a simple linear interpolation:

$$
y(x)=y^{i+1} \cdot \frac{x-x^{i}}{x^{i+1}-x^{i}}+y^{i} \cdot \frac{x^{i+1}-x}{x^{i+1}-x^{i}},
$$

with the variance:

$$
s^{2}(x)=\hat{d}^{2} \cdot \frac{\left(x-x^{i}\right) \cdot\left(x^{i+1}-x\right)}{x^{i+1}-x^{i}},
$$

if the point, in which the response value should be predicted, is between two measurement points: $x^{i} \leqslant x \leqslant x^{i+1}$. Now assume, the point, in which the response value should be predicted, is out of the interval $\left[x^{1}, x^{K}\right]$. For instance, let $x>x^{K}$. Then the predictor is coincident with the closest observed value: $y(x)=y^{K}$, and the variance of the predictor depends on the distance from this point: $s^{2}(x)=2 \cdot \hat{d}^{2} \cdot\left(x-x^{K}\right), x \geqslant x^{K}$.

The next example illustrates how the kriging procedure of a stationary process tends to that of a locally stationary process with independent increments, considered above.

EXAMPLE 3. Let us consider a stationary process with the exponential correlation function $\rho(\tau)=e^{-\frac{\tau}{\gamma}}$, mean $\mu$ and variance $\sigma^{2}=d^{2} \cdot \gamma$. Assume the points of observations to be arranged in an increasing order: $-1 \leqslant x^{1}<x^{2}<\cdots<x^{K} \leqslant 1$.

The probabilistic model considered is a well-known Ornstein-Uhlenbeck process (Gillespi, 1996). Denote $\rho_{i}=e^{-\frac{x_{i}-x_{i-1}}{\gamma}}=1-\frac{x_{i}-x_{i-1}}{\gamma}+\mathrm{O}\left(\gamma^{-2}\right)$. So MLE of the Ornstein-Uhlenbeck process parameters (Valdivieso, 2008) are approximated as follows:

$$
\begin{aligned}
\hat{\mu} & =\frac{y_{1}+\sum_{i=2}^{K} \frac{y_{i}-\rho_{i} \cdot y_{i-1}}{1+\rho_{i}}}{1+\sum_{i=2}^{K} \frac{1-\rho_{i}}{1+\rho_{i}}}=\frac{y_{1}+y_{K}}{2}+\mathrm{O}\left(\gamma^{-1}\right) \\
\hat{d}^{2} & =\hat{\sigma}^{2} / \gamma=\frac{1}{K \cdot \gamma} \cdot\left(\left(y_{1}-\hat{\mu}\right)^{2}+\sum_{i=2}^{N} \frac{\left(y_{i}-\hat{\mu}-\rho_{i} \cdot\left(y_{i-1}-\hat{\mu}\right)\right)^{2}}{1-\rho_{i}^{2}}\right) \\
& =\frac{1}{2 \cdot K} \sum_{i=2}^{K} \frac{(y-y)^{2}}{x_{i}-x_{i-1}}+\mathrm{O}\left(\gamma^{-1}\right)
\end{aligned}
$$

which correspond to (23) and (24). After simple manipulations one can make sure that the kriging predictor and its variance are approximated in a similar way:

$$
\begin{aligned}
y_{i}(x) & =\hat{\mu}+\frac{\left(y_{i}-\hat{\mu}\right) \cdot\left(\rho_{*, i-1}-\rho_{i} \cdot \rho_{i, *}\right)+\left(y_{i}-\hat{\mu}\right) \cdot\left(\rho_{i, *}-\rho_{i} \cdot \rho_{*, i-1}\right)}{1-\left(\rho_{i}\right)^{2}} \\
& =y^{i+1} \cdot \frac{x-x^{i}}{x^{i+1}-x^{i}}+y^{i} \cdot \frac{x^{i+1}-x}{x^{i+1}-x^{i}}+\mathrm{O}\left(\gamma^{-1}\right)
\end{aligned}
$$




$$
\begin{aligned}
\sigma^{2}(x) & =\hat{d}^{2} \cdot \frac{1+\left(\rho_{i}\right)^{2}-\left(\rho_{*, i-1}\right)^{2}-\left(\rho_{i, *}\right)^{2}}{1-\left(\rho_{i}\right)^{2}} \\
& =\hat{d}^{2} \cdot \frac{\left(x-x^{i}\right) \cdot\left(x^{i+1}-x\right)}{x^{i+1}-x^{i}}+O\left(\gamma^{-1}\right),
\end{aligned}
$$

that corresponds to (25), (26).

Suppose further that the points of measurement are obtained from the bounded set of homogeneity and isotropy $D$. Omitting higher order terms in (16) and taking, for simplicity, $a=1$ the correlation function is obtained:

$$
\rho(\tau)=1-\frac{\tau^{\delta}}{\gamma^{\delta}}
$$

Let us show that the function introduced can be applied to correlation modeling among measurements (9) performed at points (8) under the appropriated value of parameter $\gamma$.

Theorem 3. Let the set of vectors $X=\left(x^{1}, x^{2}, \ldots, x^{K}\right), x^{i} \in D \subset \Re^{n}, 1 \leqslant i \leqslant K$ be given. If the matrix of degrees of pairwise distances between the points of measurement $A=\left[r_{i j}^{\delta}\right]_{1}^{K}, 0<\delta \leqslant 2, r_{i j}=\left|x_{i}-x_{j}\right|, 1 \leqslant i, j \leqslant K$, is non-singular: $|A| \neq 0$, and $E \cdot A^{-1} \cdot E^{T} \neq 0$, then the matrix $\Sigma=E \cdot E^{T}-\frac{A}{\gamma^{\delta}}$ is positively defined, as $\gamma^{\delta}>1 / E^{T} \cdot A^{-1} \cdot E$.

The proof is given in Appendix 1.

Assume the values of measurements $Y$ be normally distributed with the mean $\mu$, the variance $\delta^{2}=d^{2} \cdot \gamma^{\delta} / a$ and the correlation function (31). The theorems proved and examples considered allow us to introduce the MLE of the parameters

$$
\begin{aligned}
& \hat{\mu}=\frac{y \cdot A^{-1} \cdot E^{T}}{E \cdot A^{-1} \cdot E^{T}} \\
& \hat{d}^{2}=\frac{1}{K} \cdot\left(\frac{\left(y^{T} \cdot A^{-1} \cdot E\right)^{2}}{E^{T} \cdot A^{-1} \cdot E}-y^{T} \cdot A^{-1} \cdot y\right),
\end{aligned}
$$

the kriging predictor:

$$
y(x)=y^{T} \cdot A^{-1} \cdot\left(\tau(x)-E \cdot \frac{E^{T} \cdot A^{-1} \cdot \tau(x)-1}{E^{T} \cdot A^{-1} \cdot E}\right)
$$

with the variance:

$$
s^{2}(x)=\hat{d}^{2} \cdot\left(\tau(x)^{T} \cdot A^{-1} \cdot \tau(x)-\frac{\left(E^{T} \cdot A^{-1} \cdot \tau(x)-1\right)^{2}}{E^{T} \cdot A^{-1} \cdot E}\right) .
$$

It is easy to make sure that the kriging predictor (34) is coincident with the values of response surface at measured points. The variance $s^{2}(x)$ derived in (35) may be used as a measure of uncertainty of the prediction, because it is smaller for the points 
close to the measured ones, tends to zero at measurement points, and increases, when the distance to measurements increases. Besides, variance (35) decreases in an arbitrary point $x$, when the number of measurements $K$ increases, i.e., the Gaussian density of $N(y(x), s(x))$ efficiently describes the hypothetic surface to be explored. It is convenient to use parametrization $\mu, d^{2}$ of the model considered instead a $\mu, \sigma^{2}$, because, in such a case, predictor (34) and variance (35) do not depend on the scaling parameter $\gamma$. Thus, an asymptotic LHIGF model is built, described only by two parameters $\mu$ and $d^{2}$, which enables us to create the kriging predictor, applicable to extrapolation by means of the matrix of degrees of distances between the measurement points.

EXAMPLE 4. Let us consider set (8) displayed on $K$ vertices of a regular simplex, the lengths of edges of which are equal to $r$, and the values of the response function on vertices of the simplex are $y_{1}, y_{2}, y_{K}$. Assume, for simplicity $\delta=1$.

Now the distance matrix has a simple shape: $A=r \cdot H, H=\left[H_{i j}\right]_{1}^{K}, H_{i j}=1, i \neq$ $j, H_{i i}=0,1 \leqslant i, j \leqslant K$. It is quite obviously that the inverse of the distance matrix is of a similar shape: $A^{-1}=\left[A_{i j}^{-1}\right]_{1}^{K} A_{i j}^{-1}=\frac{1}{r \cdot(K-1)}, i \neq j, A_{i i}^{-1}=\frac{1}{r \cdot(K-1)}-\frac{1}{r}$, $1 \leqslant i, j \leqslant K$. Using (32), (33), we obtain MLE's of parameters:

$$
\hat{\mu}=\frac{\sum_{i=1}^{K} y_{i}}{K}, \quad \hat{d}^{2}=\frac{\sum_{i=1}^{K}\left(y_{i}-\hat{\mu}\right)^{2}}{K} .
$$

Thus, the kriging predictor (34) becomes:

$$
y(x)=\hat{\mu}-\sum_{i=1}^{K}\left(y_{i}-\hat{\mu}\right) \cdot \frac{\tau_{i}(x)}{r},
$$

the variance (35) of which is:

$$
s^{2}(x)=\hat{d}^{2} \cdot\left(\sum_{i=1}^{K} \tau_{i}^{2}(x)-\frac{\left(\sum_{i=1}^{K} \tau_{i}^{2}(x)\right)^{2}}{K}-\frac{2 \cdot r \cdot \sum_{i=1}^{K} \tau_{i}^{2}(x)}{K}+\frac{(K-1) \cdot r^{2}}{K}\right),
$$

here $\tau(x)=\left(\tau_{1}, \tau_{2}, \ldots, \tau_{K}\right)^{T}, \tau_{i}=\left|x_{i}-x\right|, 1 \leqslant i \leqslant K$, is the vector of distances between a certain point $x \in \Re^{K}$ and the simplex vertices.

\section{Extrapolation of Test Surfaces by LHIGF Kriging Predictor}

Let us consider two examples of extrapolation of surfaces given by multivariate functions using LHIGF kriging procedure.

EXAMPLE 5. Let hypothetic surface be derived with the expression:

$$
f(x, y)=\sqrt{(x-5)^{2}+(y-5)^{2}} .
$$

The shape of this function is depicted on Fig. 1. 


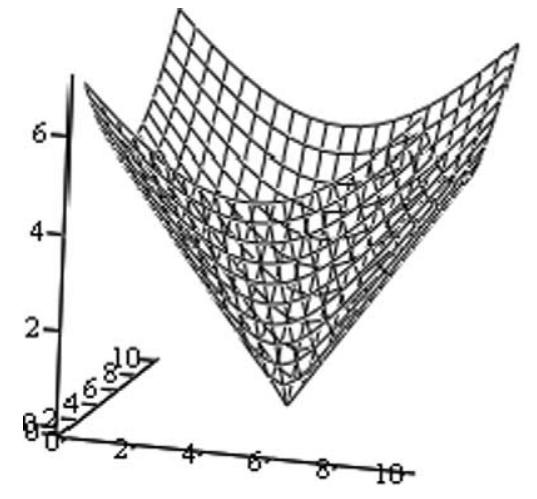

Fig. 1. Hypothetic surface (36).

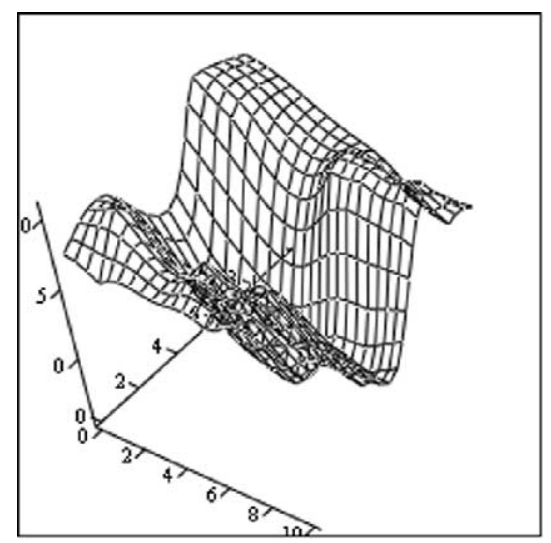

Fig. 3. Hypothetic surface (37).

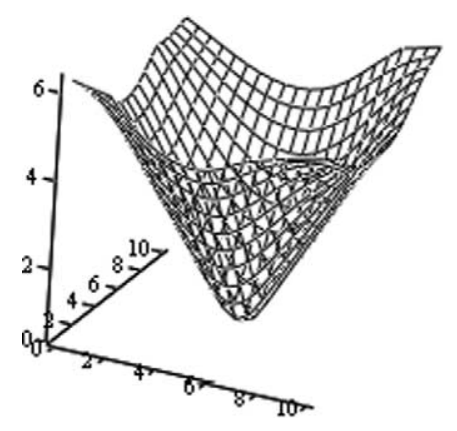

Fig. 2. Extrapolated surface with kriging.

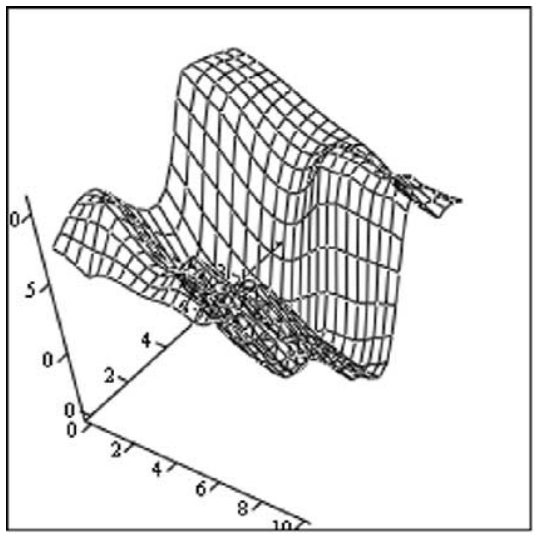

Fig. 4. Extrapolated surface by kriging.

Let the values of function (36) be computed at points, randomly and uniformly distributed on the square $\left[0 \leqslant x_{1} \leqslant 10,0 \leqslant x_{2} \leqslant 10\right], N=50$ (see Table 1 in Appendix 2). The extrapolation by the kriging predictor considered was built for this example using the MATHCAD software. MLE's of the parameters of the model are $\hat{\mu}=7.702$ and $\hat{d}^{2}=0.145$. The surface extrapolated according to conditional mean (30) is illustrated on Fig. 2. A conditional probability density can be created, too. For example, suppose $y(x)=0.445$ and $d(x)=0.255$ for $x=5, y=5$. Then the respective probabilistic model is $N(0.445,0.255)$.

EXAMPLE 6. Let the next hypothetic surface with several local extremes, be derived by the expression:

$$
f(x, y)=5 \sin (x)+\cos (y)+\sqrt{(x-5)^{2}+(y-5)^{2}} .
$$

The shape of this function is presented in Fig. 3. 
The values of the function given in (33) were computed at the points, randomly and uniformly distributed on the square $\left[0 \leqslant x_{1} \leqslant 10,0 \leqslant x_{2} \leqslant 10\right], N=50$ (see Table 2 in Appendix 2). The kriging predictor was constructed for this example by the considered approach using the MATHCAD software. MLE's of the parameters of the model are $\hat{\mu}=8.802$ and $\hat{d}^{2}=3.618$. The surface extrapolated according to conditional mean predictor (30) is depicted in Fig. 4.

Hence, the results of computer simulation show the applicability of the LHIGF extrapolator as a probabilistic model for surfaces defined by multivariate functions.

\section{Estimation of a Spacecraft Re-Entry}

In addition, the considered approach has been applied in the approximation of the position of a spacecraft entering the atmosphere. The computer code was used for data generation, which simulates the fall down position on the Earth surface of a spacecraft re-entering the atmosphere (http: // scmsa.eu/).

Details of the re-entry model are as follows. A spacecraft is a hollow sphere of $50 \mathrm{~cm}$ diameter and one centimetre thick. The sphere is made of steel (volumic mass: $8030 \mathrm{~kg} / \mathrm{m}^{3}$ ). Its weight is $247.25 \mathrm{~kg}$ and its surface is $1.57 \mathrm{~m}^{2}$. The re-entry of the spacecraft into atmosphere takes place at the altitude of around $119,330 \mathrm{~m}$ from the Earth surface. The model works in a fixed Coordinates Reference System $(X, Y, Z)$ related to stars. The Reference System centre is at the Earth centre. The $Z$ axis corresponds to the South pole - North pole axis. The $X$ axis is in the Equator plane and is directed to the Greenwich meridian. The $Y$ axis is in the Equator plane and is orthogonal to the two other axes.

The model input parameters are:

- the spacecraft coordinates at the re-entry: $X, Y, Z$ (in meters);

- the spacecraft velocity at the re-entry given by: $V_{x}, V_{y}, V_{z}$ (in $\left.\mathrm{m} / \mathrm{s}\right)$;

- the atmosphere density columns (one density value $/ 1 \mathrm{~km}$ ): Density in $\mathrm{kg} / \mathrm{m}^{3}$;

- the aerodynamic coefficient of the Spacecraft: $C_{x}$;

- the model calculates spacecraft coordinates and spacecraft velocity on the base of a 0.01 second iteration program.

The model works with the following input parameters:

- the spacecraft coordinates at the re-entry:

- $[X, X+1000 \mathrm{~m}, X-1000 \mathrm{~m}]$

- $[Y, Y+1000 \mathrm{~m}, Y-1000 \mathrm{~m}]$;

- $[Z, Z+1000 \mathrm{~m}, Z-1000 \mathrm{~m}]$;

- the spacecraft velocity at the re-entry given by $V_{x}, V_{y}, V_{z}$ :

- $\left[V_{x}, V_{x}+10 \%, V_{x}-10 \%\right]$;

- $\left[V_{y}, V_{y}+10 \%, V_{y}-10 \%\right]$

- $\left[V_{z}, V_{z}+10 \%, V_{z}-10 \%\right]$

- the atmosphere density columns (one density value $/ 1 \mathrm{~km}$ ): 
- [Density];

- [Density - 10\%];

- [Density - 5\%];

- [Density $+5 \%$ ];

- [Density $+10 \%]$;

- the aerodynamic coefficient of the spacecraft:

- $\left[C_{x}\right]$;

- $\left[C_{x}-10 \%\right]$

- $\left[C_{x}+10 \%\right]$.

The numerical values of the parameters are:

- the spacecraft coordinates at the re-entry:

- $X=[280,000,281,000,279,000]$ (three possible choices);

- $Y=[4,800,000,4,801,000,4,799,000]$ (three possible choices);

- $Z=[4,370,000,4,371,000,4,369,000]$ (three possible choices);

- the spacecraft velocity at the re-entry given by $V_{x}, V_{y}, V_{z}$ :

- $V_{x}=[2800,3080,2520]$;

- $V_{y}=[1300,1430,1170]$

- $V_{z}=[-4500,-4950,-4050]$;

- the aerodynamic coefficient of the spacecraft:

- $[0.5]$;

- $[0.45]$

- $[0.55]$.

The result of the computer simulation is the distance in meters between the projected position on Earth of the spacecraft at the time ( $T=0$ seconds) of the entry into the atmosphere and the final fall-down position of the spacecraft on Earth at the end of the simulation. By crossing all of the combinations of the parameters, 10,935 simulations have been obtained in total (http://scmsa.eu/), 90 simulations have been chosen randomly out of these 10,935, that are given in Table 3 of Appendix 2.

The kriging predictor (32) of the spacecraft re-entering distance was constructed by the considered approach. The estimated parameters of the model are as follows: $\mu=$ $252,560, d^{2}=228,795$. Next 10 values of simulation were used to evaluate the accuracy of the method. In two last rows of Table 4 of Appendix 2 measured and approximated values of spacecraft re-entering distance are given. The ratio of the standard error to the average of the approximated value is about $0.5 \%$.

\section{Conclusions}

A probabilistic model with a locally homogeneous and isotropic random field is proposed to display multivariate data using the matrix of degrees of pairwise distances among the points of observation or measurement. The model developed allows us to represent the information obtained from any number of measures of the objective function in a computational code or a physical experiment. This information can be "stored" as a density 
of probability around each point in the configuration space. The kriging procedure is built in the paper using only precise information that some measurements gave some values, and this precise value propagated as a probabilistic law. The kriging predictor developed allows prediction (values in the future) or reconstruction of the missing data (values in the past). The resulting model is rather simple and depends only on the mean and variance parameters of the probabilistic model, which are efficiently estimated by the maximal likelihood method. Application of the kriging procedure developed for extrapolation of two analytically computed surfaces and estimation of the position of the spacecraft reentering the atmosphere illustrates the applicability of the proposed probabilistic model of multivariate surfaces. Indeed, the model constructed might be generalised to a multimodal case and noisy measurements.

\section{References}

Adler, R.J. (1981). The Geometry of Random Fields. Wiley, Chichester.

Bartkute, V., Sakalauskas, L. (2009). Statistical inferences for termination of Markov type random search algorithms. Journal of Optimization Theory and Applications, 141(3), 475-493.

Beauzamy, B. (2004). Methodes probabilistes pour l'etude des phenomenes reels. Ouvrage edite par la Societe de Calcul Mathematique. http: / /www.scmsa.eu/RMM/HS_e.pdf.

Bisgaard, M.E., Sasvari, Z. (2000). Characteristic Functions and Moment Sequences: Positive Definiteness in Probability. Nova Science Publishers.

Bogush, A.J., Elkins, R.E. (1986). Gaussian field expansions for large aperture antennas. IEEE Transactions on Antennas and Propagation, AP-34(2).

Jones, D.R. (2001). A taxonomy of global optimization methods based on response surfaces. Journal of Global Optimization, 21, 345-383.

Chamon, C.C., Mudry, C., Wen. X.G. (1996). Localization in two dimensions, Gaussian field theories, and multifractality. Physical Review Letters, 77, 4194-4197.

Gillespie (1996). Exact numerical simulation of the Ornstein-Uhlenbeck process and its integral. Physical Revue E, 54, 2084-91.

Goff, J.A., Jordan, T.H. (1988). Stochastic modeling of seafloor morphology: inversion of sea beam data for second-order statistics. Journal of Geophysical Research, 93, 13589-13608.

Goff, J.A., Holliger, K., Levander, A. (1994). Model fields: a new method for characterization of random seismic velocity heterogeneity. Geophysical Research Letters, 21, 493-496.

Gower, J.C. (1982). Euclidean distance geometry. Mathematical Scientist, 7, 1-14.

Horn, R.A., Johnson, C.R. (1991). Topics in Matrix Analysis. Cambridge University Press, Cambridge.

Krige, D.G. (1951). A Statistical Approach to Some Mine Valuations and Allied Problems at the Witwatersrand. Master's thesis of the University of Witwatersrand, Australia.

Levenson, M., Rahn, F. (1981). Realistic estimates of the consequences of nuclear accidents. Nuclear Technology, 53, 99-110.

Lim, S.C., Teo, L.P. (2009). Generalized Whittle-Mattern random field as a model of correlated fluctuations. Journal of Physics, 42(10), 1-22.

Lopez-Caballero, F., Modaressi-Farahmand-Razavi, A. (2010). Assessment of variability and uncertainties effects on the seismic response of a liquefiable soil profile. Soil Dynamics and Earthquake Engineering, 30(7), $600-613$.

Matheron, G. (1963). Principles of geostatistics. Economic Geology, 58, 1246-1266.

Mattern, B. (1986). Spatial Variation. Springer, Berlin.

Mockus, J. (1968). Multiextremal Problems in Design. Nauka, Moscow (in Russian).

Mockus, J. (1989). Bayesian Approach to Global Optimization, Kluwer, Amsterdam.

Mockus, J., Eddy, W., Reklaitis, G. (1997). Bayesian Heuristic Approach to Discrete and Global Optimization. Academic Press, San Diego. 
Pottgoff, J. (2010). Sampling properties of random fields. Communications on Stochastic Analysis, 4(3), 335353.

Schumaker L. (1976). Fitting surfaces to scattered data. In: Chui, C., Schumaker, L., Lorentz, G. (Eds.), Approximation Theory II, Wiley, New York, pp. 203-268.

Shepard, D. (1968). A two dimensional interpolation function for irregularly spaced data. In: Procedings of ACM 23rd Nat'l Conference, pp. 517-524.

Solomon, K.R., Brock, T.C.M., Zwart, D., Dyer, S.D., Leo Posthuma, L., Richards, S., Sanderson, H., Sibley, P., Brink, P.J. (2008). Extrapolation Practice for Ecotoxicological Effect Characterization of Chemicals. CRC Press/Francis \& Taylor, London.

Stein, M.L. (1999). Interpolation of Spatial Data: Some Theory for Kriging. Springer, Berlin.

Stein, M.L., Chi, Z., Welty, L.J. (2004). Approximating likelihoods for large spatial data sets. Journal of the Royal Statistical Society, 66(2), 275-296.

Valdivieso, L., Schoutens, W, Tuerlinckx, F. (2008). Maximum likelihood estimation in processes of OrnsteinUhlenbeck type. Statistical Inference of Stochastic Processes, 12, 1-19.

Zaichik, L.I., Alipchenkov, V.M. (2010). Modelling of transport and dispersion of arbitrary-density particles in turbulent flows. International Journal of Heat and Fluid Flow, 31(5), 850-861.

Zilinskas, A. (1982). Axiomatic approach to statistical models and their use in multimodal optimization theory. Mathematical Programming, 22, 104-116.

\section{Appendix 1}

Proof of Theorem 1. Note that the matrix $A$ is invertible. Besides, $E \cdot A^{-1} \cdot E^{T}>0$ according to Gower (1982). Next, one can make sure by means of inversion formula (20) that

$$
\begin{aligned}
y^{T} & \left(E \cdot E^{T}-\frac{a \cdot A}{\gamma^{\delta}}+\mathrm{O}\left(\gamma^{-2 \cdot \delta}\right)\right)^{-1} \cdot E \\
& =\frac{\gamma^{\delta}}{a} \cdot\left(y^{T}\left(\frac{A^{-1} \cdot E \cdot E^{T} \cdot A^{-1}}{E^{T} \cdot A^{-1} \cdot E-a / \gamma^{\delta}}-A^{-1}\right) \cdot E+\mathrm{O}\left(\gamma^{-2 \cdot \delta}\right)\right) \\
& =\frac{\gamma^{\delta}}{a} \cdot\left(\frac{Y^{T} \cdot A^{-1} \cdot E}{E^{T} \cdot A^{-1} \cdot E-a / \gamma^{\delta}}+\mathrm{O}\left(\gamma^{-\delta}\right)\right) .
\end{aligned}
$$

Similarly,

$$
\begin{aligned}
& E^{T}\left(E \cdot E^{T}-\frac{a \cdot A}{\gamma^{\delta}}+\mathrm{O}\left(\gamma^{-2 \cdot \delta}\right)\right)^{-1} \cdot E \\
& \quad=\frac{\gamma^{\delta}}{a} \cdot\left(\frac{E^{T} \cdot A^{-1} \cdot E}{E^{T} \cdot A^{-1} \cdot E-a / \gamma^{\delta}}+\mathrm{O}\left(\gamma^{-\delta}\right)\right)
\end{aligned}
$$

Combining both these expressions with (11) estimate (18) is obtained.

Estimate (19) is obtained in a similar way after some elementary manipulations using (12), (20).

Proof of Theorem 2. According to theorem assumptions and Gower (1982) the matrix $A$ is invertible and $E \cdot A^{-1} \cdot E^{T}>0$. It follows by means of inversion formula (20) that 


$$
\begin{aligned}
1- & \tau(x)^{T} \cdot \Sigma^{-1} \cdot E \\
= & 1-\left(E-\frac{a \cdot \tau(x)}{\gamma^{\delta}}\right) \cdot\left(E \cdot E-\frac{a \cdot A}{\gamma^{\delta}}\right)^{-1} \cdot E \\
& +\mathrm{O}\left(\frac{\tau(x)^{\delta}}{\gamma^{\delta}}\right) \\
= & \frac{\gamma^{\delta}}{a} \cdot\left(\frac{\tau(x)^{T} \cdot A^{-1} \cdot E-1}{E^{T} \cdot A^{-1} \cdot E-a / \gamma^{\delta}}+\mathrm{o}\left(\frac{|\tau(x)|^{\delta}}{\gamma^{\delta}}\right)\right) .
\end{aligned}
$$

Similarly, after elementary manipulations by virtue of (20) one can obtain that:

$$
\begin{aligned}
1- & \tau(x)^{T} \cdot \Sigma^{-1} \cdot \tau(x) \\
= & 1-\left(E-\frac{a \cdot \tau(x)}{\gamma^{\delta}}\right)^{T} \cdot\left(E \cdot E^{T}-\frac{a \cdot A}{\gamma^{\delta}}\right)^{-1} \cdot\left(E-\frac{a \cdot \tau(x)}{\gamma^{\delta}}\right) \\
& +\mathrm{O}\left(\frac{|\tau(x)|^{2 \cdot \delta}}{\gamma^{2 \cdot \delta}}\right) \\
= & \frac{\gamma^{\delta}}{a} \cdot\left(\frac{\left(E^{T} \cdot A^{-1} \cdot \tau(x)-1\right)^{2}}{E^{T} \cdot A^{-1} \cdot E-a / \gamma^{\delta}}-\tau(x)^{T} \cdot A^{-1} \cdot \tau(x)\right. \\
& \left.+\mathrm{O}\left(\frac{|\tau(x)|^{\delta}}{\gamma^{\delta}}\right)\right) .
\end{aligned}
$$

Afterwards, (22) follows by means of (12), (20), (1A), (2A), (3A) and (4A). Formula (21) is proved in a similar way.

Proof of Theorem 3. Let us consider the exponential correlation function $\rho(\tau)=$ $e^{-\left(\frac{\tau}{\gamma}\right)^{\delta}}=1-\left(\frac{\tau}{\gamma}\right)^{\delta}+\mathrm{O}\left(\frac{\tau}{\gamma}\right)^{2 \cdot \delta}$. It is well-known that this function is positively defined (1986), hence, the corresponding covariance matrix of correlations among the points of set $X$, and its inverse, defined by (20), will be non-negatively defined, too. Note, according to theorem assumptions and Gower (1982) the matrix $A$ is invertible and $E \cdot A^{-1} \cdot E^{T}>0$. Hence it follows that the matrix $\frac{A^{-1} \cdot E \cdot E^{T} \cdot A^{-1}}{E^{T} \cdot A^{-1} \cdot E}-A^{-1}$ should be non-negatively defined because the error depending on $\gamma$ can be made negligible as possible by increasing this parameter. In such a case, the matrix $\frac{A^{-1} \cdot E \cdot E^{T} \cdot A^{-1}}{E^{T} \cdot A^{-1} \cdot E-1 / \gamma^{\delta}}-A^{-1}$ will be positively defined, if $\gamma^{\delta}>1 / E^{T} \cdot A^{-1} \cdot E$. Thus, the matrix $\Sigma=E \cdot E^{T}-\frac{A}{\gamma^{\delta}}$, being the inverse of the latter matrix, should also be positively defined, if $\gamma^{\delta}>1 / E^{T} \cdot A^{-1} \cdot E$. 
Appendix 2

Table 1

Data for Example 1

\begin{tabular}{|c|c|c|c|}
\hline$\overline{\text { No }}$ & $x$ & $y$ & $f(x, y)$ \\
\hline$\overline{1}$ & 0.0126842 & 1.4752 & 6.266 \\
\hline 2 & 1.9332302 & 1.416 & 9.5464 \\
\hline 3 & 5.850061 & 6.9288 & 0.808 \\
\hline 4 & 3.503081 & 4.2655 & -0.533 \\
\hline 5 & 8.2283773 & 9.666 & 9.3566 \\
\hline 6 & 1.74129 & 1.5326 & 9.7241 \\
\hline 7 & 7.1049541 & 8.2167 & 7.1512 \\
\hline 8 & 3.0398605 & 1.9135 & 3.8281 \\
\hline 9 & 0.9141127 & 8.1718 & 8.8201 \\
\hline 10 & 1.4731341 & 1.5556 & 9.9211 \\
\hline 11 & 9.8850847 & 7.3201 & 3.6958 \\
\hline 12 & 1.1907973 & 2.7959 & 8.1034 \\
\hline 13 & 0.0892266 & 6.8224 & 6.5417 \\
\hline 14 & 5.3166416 & 7.2191 & -1.28 \\
\hline 15 & 6.0176413 & 1.2303 & 2.9265 \\
\hline 16 & 1.6624948 & 8.3466 & 9.2324 \\
\hline 17 & 4.5078589 & 5.1702 & -3.933 \\
\hline 18 & 0.570559 & 4.2621 & 6.7558 \\
\hline 19 & 7.8331917 & 9.4934 & 9.3133 \\
\hline 20 & 5.1987616 & 5.4954 & -3.181 \\
\hline 21 & 8.7596822 & 4.7172 & 6.8608 \\
\hline 22 & 9.5589983 & 8.4696 & 4.4825 \\
\hline 23 & 5.393415 & 4.5609 & -3.446 \\
\hline 24 & 4.6207383 & 9.8295 & -1.054 \\
\hline 25 & 8.6221953 & 7.3918 & 8.3823 \\
\hline 26 & 7.7965831 & 1.9601 & 8.7429 \\
\hline 27 & 9.9679564 & 8.3943 & 2.9181 \\
\hline 28 & 6.1149269 & 5.0091 & 0.57 \\
\hline 29 & 2.6621391 & 0.275 & 8.5407 \\
\hline 30 & 8.4011913 & 5.7257 & 8.5963 \\
\hline 31 & 3.7585732 & 5.3132 & -1.047 \\
\hline 32 & 6.7718677 & 8.4304 & 5.6633 \\
\hline 33 & 0.088171 & 6.576 & 6.5562 \\
\hline 34 & 2.7588723 & 8.4214 & 5.4198 \\
\hline 35 & 5.8791172 & 1.0995 & 2.4866 \\
\hline 36 & 8.3760762 & 3.1409 & 7.188 \\
\hline 37 & 4.8493058 & 2.8608 & -3.77 \\
\hline 38 & 7.4372767 & 1.4028 & 9.0845 \\
\hline 39 & 4.5797574 & 8.3462 & -2.056 \\
\hline 40 & 7.4441859 & 6.0024 & 8.1886 \\
\hline 41 & 5.990413 & 2.5272 & 0.4036 \\
\hline 42 & 7.3500394 & 0.0162 & 10.888 \\
\hline 43 & 5.7239872 & 8.0624 & 0.2874 \\
\hline 44 & 1.5155716 & 2.1057 & 9.0123 \\
\hline 45 & 4.2516488 & 5.5319 & -2.83 \\
\hline 46 & 5.1712005 & 1.1378 & -0.197 \\
\hline 47 & 7.5153642 & 7.5222 & 8.6039 \\
\hline 48 & 1.6899589 & 5.4343 & 8.9638 \\
\hline 49 & 4.9188426 & 4.3671 & -4.594 \\
\hline 50 & 6.9975295 & 6.9621 & 6.8539 \\
\hline
\end{tabular}

Table 2

Data for Example 2

\begin{tabular}{llll}
\hline No & $x$ & $y$ & $f(x, y)$ \\
\hline 1 & 0.0127 & 1.4752 & 6.1072 \\
2 & 1.9332 & 1.416 & 4.717 \\
3 & 5.8501 & 6.9288 & 2.1078 \\
4 & 3.5031 & 4.2655 & 1.6674 \\
5 & 8.2284 & 9.666 & 5.674 \\
6 & 1.7413 & 1.5326 & 4.7584 \\
7 & 7.105 & 8.2167 & 3.8442 \\
8 & 3.0399 & 1.9135 & 3.6563 \\
9 & 0.9141 & 8.1718 & 5.1725 \\
10 & 1.4731 & 1.5556 & 4.9297 \\
11 & 9.8851 & 7.3201 & 5.408 \\
12 & 1.1908 & 2.7959 & 4.4009 \\
13 & 0.0892 & 6.8224 & 5.238 \\
14 & 5.3166 & 7.2191 & 2.2416 \\
15 & 6.0176 & 1.2303 & 3.9047 \\
16 & 1.6625 & 8.3466 & 4.7264 \\
17 & 4.5079 & 5.1702 & 0.5207 \\
18 & 0.5706 & 4.2621 & 4.4905 \\
19 & 7.8332 & 9.4934 & 5.312 \\
20 & 5.1988 & 5.4954 & 0.5338 \\
21 & 8.7597 & 4.7172 & 3.7703 \\
22 & 9.559 & 8.4696 & 5.7291 \\
23 & 5.3934 & 4.5609 & 0.5895 \\
24 & 4.6207 & 9.8295 & 4.8444 \\
25 & 8.6222 & 7.3918 & 4.3406 \\
26 & 7.7966 & 1.9601 & 4.1306 \\
27 & 9.968 & 8.3943 & 6.0168 \\
28 & 6.1149 & 5.0091 & 1.115 \\
29 & 2.6621 & 0.275 & 5.2718 \\
30 & 8.4012 & 5.7257 & 3.4778 \\
31 & 3.7586 & 5.3132 & 1.2803 \\
32 & 6.7719 & 8.4304 & 3.8609 \\
33 & 0.0882 & 6.576 & 5.1585 \\
34 & 2.7589 & 8.4214 & 4.09 \\
35 & 5.8791 & 1.0995 & 3.9984 \\
36 & 8.3761 & 3.1409 & 3.8541 \\
37 & 4.8493 & 2.8608 & 2.1445 \\
38 & 7.4373 & 1.4028 & 4.3451 \\
39 & 4.5798 & 8.3462 & 3.3724 \\
40 & 7.4442 & 6.0024 & 2.6418 \\
41 & 5.9904 & 2.5272 & 2.6638 \\
42 & 7.35 & 0.0162 & 5.5101 \\
43 & 5.724 & 8.0624 & 3.1468 \\
44 & 1.5156 & 2.1057 & 4.5297 \\
48 & 4.2516 & 5.5319 & 0.9181 \\
50 & 5.1712 & 1.1378 & 3.866 \\
\hline & 7.5154 & 7.5222 & 3.5621 \\
49 & 4.9188 & 4.3671 & 0.6381 \\
4.9975 & 6.9621 & 2.8 \\
\hline 15 & & &
\end{tabular}


Table 3

90 results of simulation and the set of 90 input parameters used by the spacecraft re-entry model

\begin{tabular}{|c|c|c|c|c|c|c|c|c|c|}
\hline Runs & $\begin{array}{l}X \\
(\mathrm{~m})\end{array}$ & $\begin{array}{l}Y \\
(\mathrm{~m})\end{array}$ & $\begin{array}{l}Z \\
(\mathrm{~m})\end{array}$ & $\begin{array}{l}V_{x} \\
(\mathrm{~m} / \mathrm{s})\end{array}$ & $\begin{array}{l}V_{y} \\
(\mathrm{~m} / \mathrm{s})\end{array}$ & $\begin{array}{l}V_{z} \\
(\mathrm{~m} / \mathrm{s})\end{array}$ & $C_{x}$ & $\begin{array}{l}\text { Density } \\
\text { variation } \\
(\%)\end{array}$ & $\begin{array}{l}\text { Distance between } \\
\text { projected starting } \\
\text { position and fall } \\
\text { down position }(\mathrm{m})\end{array}$ \\
\hline 1 & 281,000 & $4,799,000$ & $4,370,000$ & 2520 & 1170 & -4950 & 0.45 & 10 & $212,092.78$ \\
\hline 2 & 281,000 & $4,801,000$ & $4,370,000$ & 2800 & 1300 & -4950 & 0.5 & 0 & $233,397.68$ \\
\hline 3 & 280,000 & $4,799,000$ & $4,370,000$ & 2800 & 1300 & -4050 & 0.55 & 5 & $262,457.25$ \\
\hline 4 & 281,000 & $4,799,000$ & $4,371,000$ & 2520 & 1300 & -4050 & 0.45 & -10 & $259,118.97$ \\
\hline 5 & 279,000 & $4,801,000$ & $4,369,000$ & 3080 & 1430 & -4050 & 0.55 & -10 & $295,715.71$ \\
\hline 6 & 279,000 & $4,800,000$ & $4,370,000$ & 2520 & 1300 & -4950 & 0.45 & 10 & $224,251.73$ \\
\hline 7 & 279,000 & $4,799,000$ & $4,371,000$ & 2520 & 1170 & -4950 & 0.55 & 10 & $210,527.04$ \\
\hline 8 & 280,000 & $4,800,000$ & $4,369,000$ & 3080 & 1170 & -4050 & 0.5 & 0 & $260,709.71$ \\
\hline 9 & 279,000 & $4,800,000$ & $4,369,000$ & 2520 & 1300 & -4500 & 0.45 & 0 & $238,237.03$ \\
\hline 10 & 279,000 & $4,800,000$ & $4,369,000$ & 3080 & 1430 & -4950 & 0.45 & 10 & $250,269.93$ \\
\hline 11 & 281,000 & $4,801,000$ & $4,370,000$ & 2800 & 1430 & -4050 & 0.55 & -5 & $284,815.18$ \\
\hline 12 & 280,000 & $4,801,000$ & $4,369,000$ & 2800 & 1300 & -4050 & 0.45 & -10 & $270,292.55$ \\
\hline 13 & 279,000 & $4,800,000$ & $4,371,000$ & 2800 & 1170 & -4500 & 0.5 & 10 & $234,038.95$ \\
\hline 14 & 280,000 & $4,800,000$ & $4,369,000$ & 2800 & 1430 & -4950 & 0.5 & 0 & $242,122.46$ \\
\hline 15 & 280,000 & $4,799,000$ & $4,369,000$ & 2520 & 1300 & -4500 & 0.55 & -10 & $235,315.76$ \\
\hline 16 & 281,000 & $4,799,000$ & $4,371,000$ & 2800 & 1300 & -4050 & 0.45 & 5 & $267,431.27$ \\
\hline 17 & 279,000 & $4,800,000$ & $4,370,000$ & 2800 & 1170 & -4950 & 0.5 & -10 & $221,753.08$ \\
\hline 18 & 280,000 & $4,800,000$ & $4,370,000$ & 2800 & 1170 & -4050 & 0.55 & -10 & $251,519.25$ \\
\hline 19 & 279,000 & $4,800,000$ & $4,371,000$ & 2520 & 1170 & -4050 & 0.45 & -10 & $245,512.17$ \\
\hline 20 & 279,000 & $4,800,000$ & $4,369,000$ & 3080 & 1300 & -4050 & 0.45 & -5 & $278,851.77$ \\
\hline 21 & 280,000 & $4,799,000$ & $4,370,000$ & 2520 & 1430 & -4050 & 0.45 & -5 & $272,908.62$ \\
\hline 22 & 279,000 & $4,799,000$ & $4,369,000$ & 2800 & 1300 & -4500 & 0.45 & 5 & $244,501.31$ \\
\hline 23 & 279,000 & $4,799,000$ & $4,370,000$ & 2520 & 1300 & -4500 & 0.45 & 5 & $237,277.62$ \\
\hline 24 & 279,000 & $4,801,000$ & $4,370,000$ & 3080 & 1430 & -4050 & 0.5 & 5 & $296,416.81$ \\
\hline 25 & 280,000 & $4,801,000$ & $4,371,000$ & 2800 & 1300 & -4950 & 0.5 & 0 & $234,666.60$ \\
\hline 26 & 280,000 & $4,800,000$ & $4,370,000$ & 2520 & 1430 & -4500 & 0.45 & 5 & $252, \varepsilon$ \\
\hline 27 & 281,000 & $4,801,000$ & $4,370,000$ & 3080 & 1170 & -4050 & 0.5 & 0 & $264,289.84$ \\
\hline 28 & 279,000 & $4,801,000$ & $4,369,000$ & 2520 & 1170 & -4050 & 0.45 & -5 & $243,564.25$ \\
\hline 29 & 279,000 & $4,801,000$ & $4,370,000$ & 3080 & 1300 & -4950 & 0.5 & -10 & $242,623.21$ \\
\hline 30 & 279,000 & $4,799,000$ & $4,370,000$ & 3080 & 1300 & -4050 & 0.45 & -10 & $279,460.41$ \\
\hline 31 & 279,000 & $4,801,000$ & $4,371,000$ & 3080 & 1300 & -4050 & 0.5 & 5 & $280,665.20$ \\
\hline 32 & 279,000 & $4,799,000$ & $4,369,000$ & 2520 & 1430 & -4500 & 0.5 & 10 & $247,064.94$ \\
\hline 33 & 280,000 & $4,800,000$ & $4,371,000$ & 3080 & 1430 & -4500 & 0.5 & -5 & $273,419.71$ \\
\hline 34 & 281,000 & $4,800,000$ & $4,370,000$ & 2800 & 1430 & -4500 & 0.55 & -5 & $260,566.53$ \\
\hline 35 & 279,000 & $4,800,000$ & $4,370,000$ & 3080 & 1170 & -4500 & 0.45 & -10 & $246,146.34$ \\
\hline 36 & 279,000 & $4,800,000$ & $4,371,000$ & 3080 & 1300 & -4500 & 0.5 & -5 & $258,656.40$ \\
\hline 37 & 279,000 & $4,799,000$ & $4,370,000$ & 2800 & 1170 & -4500 & 0.55 & 0 & $230,969.19$ \\
\hline 38 & 280,000 & $4,800,000$ & $4,370,000$ & 2520 & 1300 & -4500 & 0.55 & -10 & $238,434.02$ \\
\hline 39 & 279,000 & $4,799,000$ & $4,370,000$ & 2520 & 1170 & -4050 & 0.5 & 10 & $237,998.19$ \\
\hline 40 & 281,000 & $4,801,000$ & $4,371,000$ & 2800 & 1170 & -4950 & 0.5 & -10 & $224,935.74$ \\
\hline 41 & 280,000 & $4,799,000$ & $4,370,000$ & 2800 & 1430 & -4950 & 0.45 & 5 & $242,704.23$ \\
\hline 42 & 281,000 & $4,799,000$ & $4,370,000$ & 2520 & 1170 & -4500 & 0.45 & -5 & $226,373.39$ \\
\hline 43 & 279,000 & $4,799,000$ & $4,369,000$ & 3080 & 1300 & -4950 & 0.5 & -10 & $237,721.87$ \\
\hline 44 & 280,000 & $4,799,000$ & $4,371,000$ & 2520 & 1300 & -4500 & 0.5 & 0 & $238,035.92$ \\
\hline 45 & 280,000 & $4,801,000$ & $4,371,000$ & 2520 & 1300 & -4950 & 0.55 & -5 & $226,603.43$ \\
\hline
\end{tabular}




\begin{tabular}{|c|c|c|c|c|c|c|c|c|c|}
\hline Runs & $\begin{array}{l}X \\
(\mathrm{~m})\end{array}$ & $\begin{array}{l}Y \\
(\mathrm{~m})\end{array}$ & $\begin{array}{l}Z \\
(\mathrm{~m})\end{array}$ & $\begin{array}{l}V_{x} \\
(\mathrm{~m} / \mathrm{s})\end{array}$ & $\begin{array}{l}V_{y} \\
(\mathrm{~m} / \mathrm{s})\end{array}$ & $\begin{array}{l}V_{z} \\
(\mathrm{~m} / \mathrm{s})\end{array}$ & $C_{x}$ & $\begin{array}{l}\text { Density } \\
\text { variation } \\
(\%)\end{array}$ & $\begin{array}{l}\text { Distance between } \\
\text { projected starting } \\
\text { position and fall } \\
\text { down position }(\mathrm{m})\end{array}$ \\
\hline 46 & 279,000 & $4,799,000$ & $4,369,000$ & 2800 & 1170 & -4500 & 0.55 & -5 & $230,281.39$ \\
\hline 47 & 281,000 & $4,801,000$ & $4,371,000$ & 2520 & 1170 & -4050 & 0.45 & 5 & $245,270.02$ \\
\hline 48 & 279,000 & $4,800,000$ & $4,370,000$ & 2520 & 1300 & -4500 & 0.55 & -10 & $238,291.58$ \\
\hline 49 & 279,000 & $4,800,000$ & $4,370,000$ & 2520 & 1170 & -4050 & 0.5 & -5 & $241,794.77$ \\
\hline 50 & 281,000 & $4,799,000$ & $4,371,000$ & 3080 & 1300 & -4050 & 0.55 & -10 & $278,121.87$ \\
\hline 51 & 279,000 & $4,800,000$ & $4,371,000$ & 2800 & 1300 & -4500 & 0.55 & -5 & $247,723.44$ \\
\hline 52 & 281,000 & $4,800,000$ & $4,369,000$ & 2800 & 1170 & -4500 & 0.5 & 0 & $232,861.02$ \\
\hline 53 & 279,000 & $4,800,000$ & $4,371,000$ & 3080 & 1300 & -4500 & 0.45 & 5 & $258,741.08$ \\
\hline 54 & 279,000 & $4,801,000$ & $4,369,000$ & 2800 & 1430 & -4500 & 0.5 & -5 & $262,041.17$ \\
\hline 55 & 281,000 & $4,800,000$ & $4,369,000$ & 2520 & 1430 & -4050 & 0.55 & -5 & $270,100.91$ \\
\hline 56 & 281,000 & $4,799,000$ & $4,371,000$ & 2520 & 1170 & -4950 & 0.5 & -10 & $214,624.18$ \\
\hline 57 & 281,000 & $4,799,000$ & $4,371,000$ & 3080 & 1430 & -4050 & 0.5 & 10 & $293,581.81$ \\
\hline 58 & 279,000 & $4,799,000$ & $4,370,000$ & 2520 & 1170 & -4500 & 0.5 & 0 & $223,967.71$ \\
\hline 59 & 279,000 & $4,799,000$ & $4,369,000$ & 2520 & 1430 & -4500 & 0.45 & -10 & $251,745.01$ \\
\hline 60 & 279,000 & $4,799,000$ & $4,369,000$ & 3080 & 1170 & -4500 & 0.5 & 5 & $239,098.46$ \\
\hline 61 & 279,000 & $4,801,000$ & $4,371,000$ & 2800 & 1170 & -4500 & 0.55 & -5 & $236,424.58$ \\
\hline 62 & 280,000 & $4,799,000$ & $4,369,000$ & 2800 & 1430 & -4050 & 0.45 & 0 & $281,537.57$ \\
\hline 63 & 281,000 & $4,801,000$ & $4,369,000$ & 3080 & 1300 & -4050 & 0.55 & 5 & $276,116.98$ \\
\hline 64 & 281,000 & $4,800,000$ & $4,371,000$ & 3080 & 1170 & -4500 & 0.55 & -10 & $244,958.44$ \\
\hline 65 & 280,000 & $4,800,000$ & $4,369,000$ & 2800 & 1170 & -4050 & 0.55 & 0 & $248,427.92$ \\
\hline 66 & 280,000 & $4,801,000$ & $4,371,000$ & 2520 & 1300 & -4500 & 0.55 & 0 & $240,026.72$ \\
\hline 67 & 280,000 & $4,801,000$ & $4,369,000$ & 2520 & 1170 & -4500 & 0.55 & 5 & $223,964.42$ \\
\hline 68 & 281,000 & $4,799,000$ & $4,371,000$ & 3080 & 1170 & -4500 & 0.55 & 10 & $240,249.89$ \\
\hline 69 & 280,000 & $4,800,000$ & $4,369,000$ & 2520 & 1300 & -4950 & 0.5 & 10 & 6 \\
\hline 70 & 280,000 & $4,801,000$ & $4,369,000$ & 2800 & 1300 & -4050 & 0.55 & 0 & $265,392.59$ \\
\hline 71 & 281,000 & $4,801,000$ & $4,370,000$ & 2800 & 1170 & -4050 & 0.5 & 0 & $253,280.62$ \\
\hline 72 & 281,000 & $4,799,000$ & $4,370,000$ & 2520 & 1170 & -4050 & 0.45 & -10 & $42,704.32$ \\
\hline 73 & 279,000 & $4,799,000$ & $4,369,000$ & 2520 & 1430 & -4050 & 0.45 & -10 & $272,016.74$ \\
\hline 74 & 280,000 & $4,799,000$ & $4,370,000$ & 2520 & 1430 & -4050 & 0.55 & 0 & $268,800.89$ \\
\hline 75 & 279,000 & $4,799,000$ & $4,371,000$ & 3080 & 1430 & -4050 & 0.45 & 10 & $295,070.17$ \\
\hline 76 & 279,000 & $4,800,000$ & $4,370,000$ & 3080 & 1430 & -4500 & 0.5 & 5 & $269,944.23$ \\
\hline 77 & 280,000 & $4,801,000$ & $4,369,000$ & 3080 & 1170 & -4500 & 0.45 & 0 & $244,998.67$ \\
\hline 78 & 281,000 & $4,799,000$ & $4,369,000$ & 2800 & 1430 & -4050 & 0.5 & 0 & $279,920.43$ \\
\hline 79 & 280,000 & $4,800,000$ & $4,371,000$ & 3080 & 1430 & -4500 & 0.45 & 10 & $272,734.62$ \\
\hline 80 & 281,000 & $4,799,000$ & $4,371,000$ & 2800 & 1300 & -4050 & 0.5 & 0 & $266,522.79$ \\
\hline 81 & 280,000 & $4,799,000$ & $4,370,000$ & 3080 & 1430 & -4500 & 0.5 & 5 & $268,191.32$ \\
\hline 82 & 281,000 & $4,801,000$ & $4,371,000$ & 2520 & 1430 & -4950 & 0.5 & 0 & $239,245.52$ \\
\hline 83 & 280,000 & $4,799,000$ & $4,370,000$ & 3080 & 1430 & -4950 & 0.55 & 0 & $248,515.87$ \\
\hline 84 & 280,000 & $4,799,000$ & $4,369,000$ & 3080 & 1170 & -4950 & 0.45 & 10 & $225,221.51$ \\
\hline 85 & 279,000 & $4,800,000$ & $4,369,000$ & 3080 & 1300 & -4950 & 0.55 & 5 & $235,766.18$ \\
\hline 86 & 280,000 & $4,799,000$ & $4,371,000$ & 2800 & 1300 & -4950 & 0.55 & 0 & $229,998.33$ \\
\hline 87 & 281,000 & $4,801,000$ & $4,370,000$ & 2520 & 1170 & -4500 & 0.5 & -5 & $228,123.31$ \\
\hline 88 & 280,000 & $4,799,000$ & $4,369,000$ & 2800 & 1300 & -4950 & 0.5 & -10 & $230,023.95$ \\
\hline 89 & 281,000 & $4,799,000$ & $4,369,000$ & 3080 & 1170 & -4500 & 0.5 & -10 & $241,697.05$ \\
\hline 90 & 281,000 & $4,799,000$ & $4,371,000$ & 2800 & 1300 & -4500 & 0.5 & -5 & $247,707.61$ \\
\hline
\end{tabular}


Table 4

Comparison of the simulated and approximated distance between the projected starting position and fall down position

\begin{tabular}{lllllllllll}
\hline Runs & $\begin{array}{l}X \\
(\mathrm{~m})\end{array}$ & $\begin{array}{l}Y \\
(\mathrm{~m})\end{array}$ & $\begin{array}{l}Z \\
(\mathrm{~m})\end{array}$ & $\begin{array}{l}V_{x} \\
(\mathrm{~m} / \mathrm{s})\end{array}$ & $\begin{array}{l}V_{y} \\
(\mathrm{~m} / \mathrm{s})\end{array}$ & $\begin{array}{l}V_{z} \\
(\mathrm{~m} / \mathrm{s})\end{array}$ & $\begin{array}{l}C_{x} \\
\text { Density } \\
\text { variation } \\
(\%)\end{array}$ & $\begin{array}{l}\text { Distance between } \\
\text { projected starting } \\
\text { position and fall } \\
\text { down position }(\mathrm{m})\end{array}$ & $\begin{array}{l}\text { Approximated } \\
\text { distance } \\
(\mathrm{m})\end{array}$ \\
\hline 91 & 281,000 & $4,801,000$ & $4,370,000$ & 3080 & 1170 & -4950 & 0.55 & -10 & $230,026.58$ & $236,383.98$ \\
92 & 279,000 & $4,801,000$ & $4,369,000$ & 2520 & 1170 & -4050 & 0.5 & -10 & $242,826.62$ & $243,739.90$ \\
93 & 281,000 & $4,800,000$ & $4,370,000$ & 2520 & 1300 & -4950 & 0.55 & 0 & 223076.11 & 231519.18 \\
94 & 280,000 & $4,801,000$ & $4,370,000$ & 2520 & 1170 & -4950 & 0.5 & 0 & $214,876.78$ & $225,845.52$ \\
95 & 280,000 & $4,800,000$ & $4,371,000$ & 2520 & 1170 & -4500 & 0.45 & -10 & $229,920.46$ & $240,656.65$ \\
96 & 280,000 & $4,799,000$ & $4,371,000$ & 2520 & 1170 & -4050 & 0.5 & 0 & $240,949.89$ & $252,944.45$ \\
97 & 281,000 & $4,799,000$ & $4,371,000$ & 2520 & 1430 & -4500 & 0.55 & 10 & $248,935.24$ & $244,524.39$ \\
98 & 279,000 & $4,801,000$ & $4,371,000$ & 2520 & 1430 & -4500 & 0.55 & 0 & $253,660.41$ & $243,268.28$ \\
99 & 280,000 & $4,799,000$ & $4,370,000$ & 2520 & 1170 & -4950 & 0.5 & 5 & $211,200.83$ & $224,797.25$ \\
100 & 280,000 & $4,800,000$ & $4,370,000$ & 3080 & 1430 & -4050 & 0.5 & -10 & $297,289.96$ & $274,333.69$
\end{tabular}


L. Sakalauskas has graduated from the Kaunas Polytechnical Institute (1970), received the PhD degree from this Institute (1974) and the degree of doctor habilius from the Institute of Mathematics and Informatics (2000). President of the Lithuanian Operational Research Society (2010), elected member of the International Statistical Institute (2002), presently is a head of the Operational Research Sector of the Vilnius University Institute of Mathematics and Informatics and professor of the Department of Information Technologies of the Vilnius Gediminas Technical University. His research interests include stochastic modeling and optimization with applications.

\title{
Lokalieji ir izotropiniai Gauso laukai krikinge
}

\author{
Leonidas SAKALAUSKAS
}

Straipsnyje nagrinejjamas lokaliai vienalyčiu ir izotropinių Gauso laukų panaudojimas daugiamačių duomenų struktūrų tikimybiniam modeliavimui. Ištirtas asimptotinis modelis, gaunamas artinant ị begalybę koreliacijos funkcijos parametrą. Sukurta krigingo procedūra nesudètingo ekstrapoliatoriaus, priklausančio tik nuo atstumų tarp matavimo taškų trupmeninių laipsnių, pavidalu. Gautas modelis apibūdinamas tik vidurkio ir dispersijos parametrais, kurie gali būti ivvertinti didžiausio tikètinumo metodu. Pateikiami dviejų ekstrapoliavimo testinių uždavinių ir kosminio palydovo patekimo ị atmosferą apskaičiavimo rezultatai, gauti sukurtuoju metodu. 